\title{
Design and Performance Analysis of Oscillator Circuit for High-Impedance Quartz Vibrating Beam
}

\author{
Liye Zhao, Hui Yang, Jinxing Liang and Libin Huang* \\ Key Laboratory of Micro-Inertial Instrument and Advanced Navigation Technology, \\ Ministry of Education, School of Instrument Science and Engineering, Southeast University, \\ Nanjing 210096, China
}

(Received October 19, 2012; accepted May 20, 2013)

Key words: high-impedance, quartz vibrating beam, oscillator circuit, small-signal model

In this paper, the design, analysis and experimental results of the multiple commonemitter series oscillator circuit with negative feedback for a high-impedance quartz vibrating beam, which is used in a recently designed accelerometer, are presented. The equivalent circuit parameters of the quartz vibrating beam are introduced and the impedance characteristic is analyzed. For the oscillation loop analysis based on the small-signal model analysis method, the model of the oscillation loop is established and analyzed. Using the analytical results, the multiple common-emitter series oscillator circuit parameters are designed and the expected loop performance is characterized by simulations. Then the resonant system is practically implemented using the highimpedance vibrating beam and the multiple common-emitter series oscillator circuit. The resonant system performance is evaluated using the Agilent frequency meter 53132A. The experimental results show that the high-impedance quartz vibrating beam vibrates stably with the multiple common-emitter series oscillator circuit, and the vibrating frequency bias stability of the system is less than $8 \mathrm{mHz}$.

\section{Introduction}

Resonant sensors are widely used to measure physical quantities such as temperature, force, or pressure. With the development of quartz micromachining technology, vibration microbeam-based microsensors such as the force gauge, accelerometer, gyro sensor and microactuators, have drawn much attention. ${ }^{(1-3)}$ In the fabrication of these sensors, in order to simplify the fabrication process of complex-structure resonators and reduce fabrication costs, sometimes the excitation electrode area of resonators is reduced. This always leads to a significant increase in the equivalent impedance of the resonator, and the resonator does not work with a conventional oscillator circuit.

In this work, in order to design the high-performance oscillator circuit for the high-impedance quartz vibrating beam used in a recently designed accelerometer, the

${ }^{*}$ Corresponding author: e-mail: huanglibin@seu.edu.cn 
small-signal model of the system composed of the traditional dual common-emitter series oscillator and the high-impedance quartz vibrating beam circuit is established and analyzed. On the basis of the relationship between the transconductance of the circuit and the resonator impedance, additional gain links are added to the traditional dual common-emitter series oscillator circuit, which is called the multiple commonemitter series oscillator. In this way, the effect of the resonator impedance on the transconductance of the circuit is reduced, and the high-impedance quartz vibrating beam works stably with the improved circuit. Simulations and experiments show that the improved circuit achieves better performance than the traditional circuit, and the vibrating frequency bias stability of the system is less than $8 \mathrm{mHz}$.

\section{Design and Analysis of Oscillator Circuit}

\subsection{Analysis of equivalent impedance of quartz resonator}

The equivalent electrical model of the quartz resonator is shown in Fig. $1,{ }^{(4)}$ where $L_{\mathrm{q}}$ is the dynamic inductance, $C_{\mathrm{q}}$ is the dynamic capacity, $R_{\mathrm{q}}$ is the dynamic resistance, and $C_{0}$ is the static capacitance. Therefore, the admittance of the quartz crystal $Y_{\mathrm{AB}}$ can be expressed as ${ }^{(5)}$

$$
Y_{A B}=j \omega C_{0}+\frac{1}{R_{\mathrm{q}}+j \omega L_{\mathrm{q}}+\frac{1}{j \omega C_{\mathrm{q}}}}=y_{0}+y_{1},
$$

where $y_{0}=j \omega C_{0}$ and $y_{1}=\frac{1}{R_{\mathrm{q}}+j \omega L_{\mathrm{q}}+\frac{1}{j \omega C_{\mathrm{q}}}}$. Since the relatively high quality factor of quartz crystal, $y_{0}$ can be approximated by $j \omega_{\mathrm{s}} C_{0}$, where $\omega_{\mathrm{s}}$ is the series resonance angular frequency of the quartz crystal. Then the equation $y_{1}=0$ can be converted to the equation of a circle with a radius of $1 /\left(2 R_{q}\right)$ as

$$
\left[\frac{R_{\mathrm{q}}}{R_{\mathrm{q}}^{2}+\left(\omega L_{\mathrm{q}}-\frac{1}{\omega C_{\mathrm{q}}}\right)^{2}}-\frac{1}{2 R_{\mathrm{q}}}\right]+\left[\frac{-\left(\omega L_{\mathrm{q}}-\frac{1}{\omega C_{\mathrm{q}}}\right)^{2}}{R_{\mathrm{q}}^{2}+\left(\omega L_{\mathrm{q}}-\frac{1}{\omega C_{\mathrm{q}}}\right)^{2}}\right]=\left(\frac{1}{2 R_{\mathrm{q}}}\right)^{2} .
$$

The admittance circle of the quartz crystal is shown in Fig. 2, where $f_{\mathrm{s}}$ is the series
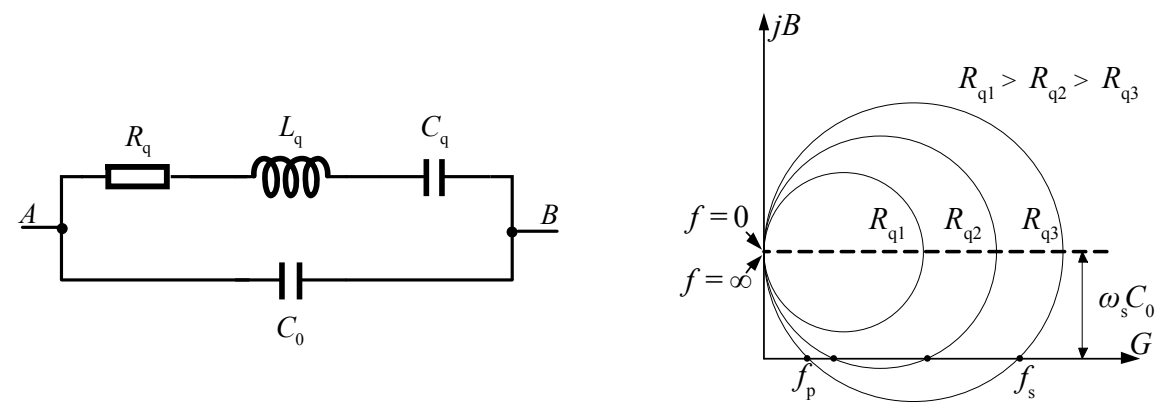

Fig. 1 (left). Equivalent electrical model of quartz resonator.

Fig. 2 (right). Quartz crystal's admittance circles. 
resonance frequency of the quartz beam, $f_{\mathrm{p}}$ is the parallel resonance frequency and $R_{\mathrm{q} 1}, R_{\mathrm{q} 2}$, and $R_{\mathrm{q} 3}$ are different dynamic resistances. The greater the crystal equivalent resistance is, the smaller the radius of the admittance circle is. The admittance circle with a small radius may have no intersection points with the horizontal axis in the coordinate grid, that is, no excitation circuit can adequately drive the quartz vibrating beam under this condition.

The test result of the parameters of the quartz vibrating beam used in this study is shown in Table 1. According to Fig. 2 and Table 1, with a high dynamic resistance and a small-radius admittance circle, this quartz beam is difficult to excite using traditional oscillator circuits. Therefore, here, we will focus on a quartz resonator with a high impedance and design an oscillator circuit that is suitable for the high-impedance resonator to vibrate steadily.

\subsection{Analysis of common-emitter series oscillator circuit}

According to the related theory of quartz resonators, ${ }^{(6,7)}$ it is more appropriate to adopt the series oscillator circuit when the quartz resonator serves as the sensitive part of sensors. Firstly, the automatic frequency-stabilization ability of the parallel resonant circuit may conceal the frequency changes caused by the factors to be measured, and this characteristic will reduce the sensitivity of the sensor. Secondly, the frequency of the parallel oscillator circuit is related to the external capacitance. It could bring about some measurement errors. In contrast, the resonance frequency of the series oscillator circuit entirely depends on the quartz vibrating beam itself and the external influence is extremely small. When the quartz beam vibrates at the series resonance frequency, the quartz beam can be equivalent to a pure resistance with the minimum impedance and relatively weak automatic frequency-stabilization ability. It has no effects on the sensitivity of the sensor. Therefore, the series resonant circuit should be adopted to design a quartz beam oscillator circuit used in the acceleration sensor. In this paper, a series oscillator circuit, which can excite the high-impedance quartz resonator, will be designed, on the basis of the analysis of the common-emitter series resonance oscillator circuit small signal model.

A kind of dual common-emitter non-sinusoidal wave series oscillator circuit is shown in Fig. 3. ${ }^{(8)}$ The transistors $\mathrm{Q}_{1}$ and $\mathrm{Q}_{2}$ offer 180 degrees phase shifts and compose a twostage common-emitter amplifier. The quartz resonator $X$ constitutes a positive feedback network. When the resonator works on the series resonant state, the impedance of the resonator is the minimum value, and the positive feedback is strongest. The outputs of the oscillator circuit are rectangular-wave signals that contain a wealth of high harmonics, so the system has multiple vibrations.

The small signal model of the circuit is shown in Fig. 4. The complex oscillation

Table 1

Equivalent parameters of the quartz beam.

\begin{tabular}{lcl}
\hline Parameters & Value & Notes \\
\hline$C_{\mathrm{q}}$ & $0.15035(\mathrm{fF})$ & Dynamic capacity \\
$R_{\mathrm{q}}$ & $2.22227(\mathrm{M} \Omega)$ & Dynamic resistance \\
$L_{\mathrm{q}}$ & $39.1341(\mathrm{kH})$ & Dynamic inductance \\
$C_{0}$ & $596.962(\mathrm{fF})$ & Static capacitance \\
$f_{\mathrm{s}}$ & $65.6132(\mathrm{kHz})$ & Series resonace frequency \\
\hline
\end{tabular}



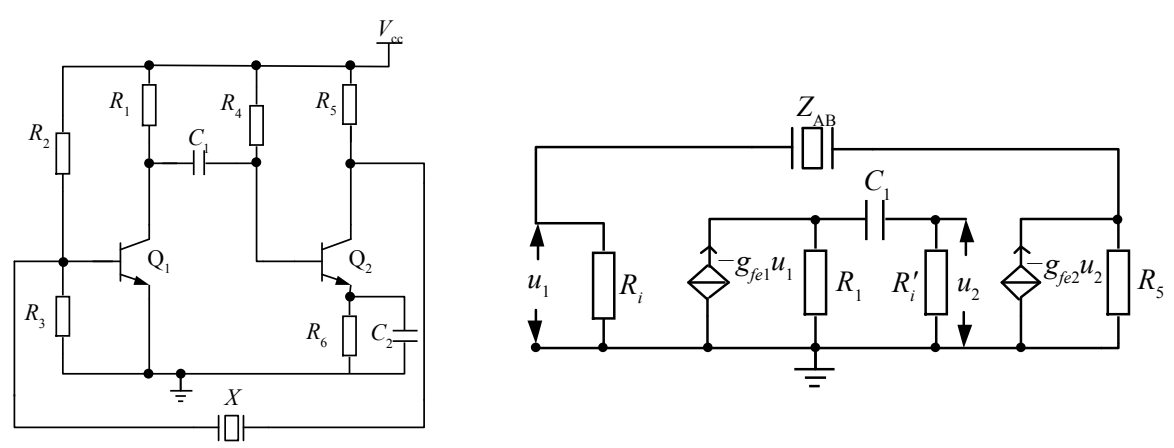

Fig. 3 (left). Dual common-emitter series oscillator circuit.

Fig. 4 (right). Small signal model of the circuit.

equation of the circuit can be deduced from the circuit working condition:

$$
-g_{f e 2}\left(-g_{f e 1} \cdot \frac{R_{1}}{\frac{1}{j \omega C_{1}}+R_{i}^{\prime}+R_{1}} \cdot R_{i}^{\prime}\right) \cdot \frac{R_{5} \cdot R_{i}}{R_{5}+Z_{\mathrm{AB}}+R_{i}}=1,
$$

where $R_{i}$ and $R_{i}{ }^{\prime}$ are the input resistances of the first-stage and second-stage transistors, respectively, $g_{f e 1}$ and $g_{f e 2}$ are the common emitter transconductances, and $Z_{\mathrm{AB}}=\frac{1}{Y_{\mathrm{AB}}}$ is the impedance of quartz. Assuming that the common emitter transconductances of the two transistors are the same, then

$$
g_{f e}=g_{f e 1}=g_{f e 2}=\frac{g_{f 0}}{1+j \frac{f}{f_{\beta}}},
$$

where $g_{f 0}$ is the low-frequency common emitter transconductance and $f_{\beta}$ is the transistor cut-off frequency. According to eq. (3), and assuming both the real and imaginary parts be zero, the circuit self-excited oscillation amplitude equilibrium condition and phase equilibrium condition are deduced.

$$
\begin{gathered}
-g_{f e}^{2} R_{1} R_{i}^{\prime} R_{5} R_{i}+\left(R_{5}+R_{i}+R_{\mathrm{q}}\right)\left(R_{i}^{\prime}+R_{1}\right)+\frac{L_{\mathrm{q}}}{C_{1}}-\frac{1}{\omega^{2} C_{1} C_{\mathrm{q}}}=0 \\
-\frac{R_{5}+R_{i}+R_{\mathrm{q}}}{C_{1}}+\left(R_{i}^{\prime}+R_{1}\right)\left(\omega^{2} L_{\mathrm{q}}-\frac{1}{C_{\mathrm{q}}}\right)=0
\end{gathered}
$$

From eq. (5),

$$
\omega=\sqrt{\frac{R_{5}+R_{i}+R_{\mathrm{q}}}{L_{\mathrm{q}} C_{1}\left(R_{i}^{\prime}+R_{1}\right)}+\frac{1}{L_{\mathrm{q}} C_{\mathrm{q}}}},
$$

where $C_{1}$ is $10 \mathrm{nF}$ and $R_{5}, R_{i}, R_{i}{ }^{\prime}$ are 1 to $3 \mathrm{k} \Omega$. Thus, $\frac{R_{5}+R_{i}+R_{\mathrm{q}}}{L_{\mathrm{q}} C_{1}\left(R_{i}^{\prime}+R_{1}\right)}<<\frac{1}{L_{\mathrm{q}} C_{\mathrm{q}}}$; then 


$$
\omega \approx \sqrt{\frac{1}{L_{\mathrm{q}} C_{\mathrm{q}}}} .
$$

That is, the circuit works at a frequency slightly higher than the series resonant frequency. Substituting eq. (8) into eq. (5), the computation formula of the minimum transistor transconductance for the circuit oscillation can be deduced to be

$$
g_{f e}=\sqrt{\frac{\left(R_{5}+R_{i}+R_{\mathrm{q}}\right)\left(R_{i}^{\prime}+R_{1}\right)}{R_{1} R_{5} R_{i} R_{i}^{\prime}}} .
$$

According to eq. (9), the computing transistor transconductance increases with the resonator equivalent impedance. In Fig. $3, R_{5}=2 \mathrm{k} \Omega, R_{i}=1 \mathrm{k} \Omega, R_{i}=1 \mathrm{k} \Omega$, and $R_{1}=6.8$ $\mathrm{k} \Omega$; then $g_{f e} \approx 0.0357 \mathrm{~S}$. However, the actual transistor transconductance is $2-10 \mathrm{~mA} / \mathrm{V}$ $(0.002-0.01 \mathrm{~S})$. Therefore, the traditional dual common-emitter series oscillator circuit cannot satisfy the starting oscillation condition for a quartz resonator with the parameters described in Table 1.

\subsection{Design of multiple common-emitter series oscillator circuit}

According to eq. (9), in order to match the computing transistor transconductance of the oscillator circuit for the high-impedance resonator to the actual transistor transconductance, the circuit shown in Fig. 3 is improved by adding an additional driving amplifier circuit and a voltage negative feedback circuit. The improved circuit is shown in Fig. 5. The improved circuit has a higher gain than the circuit shown in Fig. 3, and it is helpful for the oscillator circuit to drive the high-impedance resonator. Moreover, the additional feedback loop expands the circuit pass band, reduces the effect of factors such as power supply voltage fluctuations and temperature changes on the oscillation frequency, and increases the signal-to-noise ratio of the oscillation signal.

Assuming that the amplification factor of the added two transistor amplifier circuits for the former signal is $A \mathrm{e}^{i \theta}(A>1)$ and ideal phase shift of the two common-emitter transistor series circuit is $360 \mathrm{deg}$, then $\mathrm{e}^{i \theta}=1$. According to eq. (9), the computation equation of the minimum transistor transconductance determined by the circuit oscillation can be expressed as

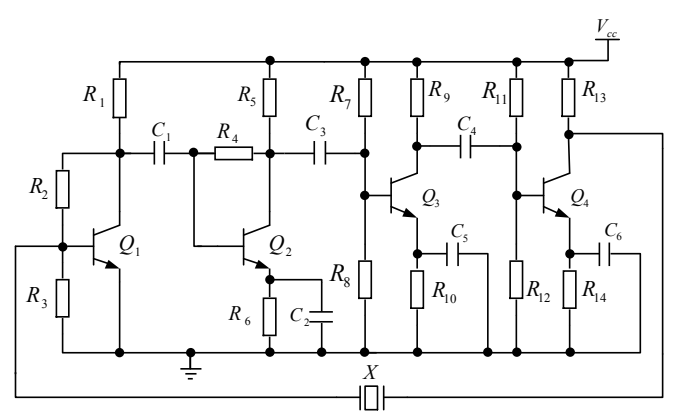

Fig. 5. Multiple common-emitter series oscillator circuit. 


$$
g_{f e}=\sqrt{\frac{\left(R_{5}+R_{i}+R_{\mathrm{q}}\right)\left(R_{i}^{\prime}+R_{1}\right)}{R_{1} R_{5} R_{i}^{\prime} R_{i} A}} .
$$

In the comparison of eq. (9) with eq. (10), since $A>1$, the minimum transconductance deduced from eq. (10) is smaller than that deduced from eq. (9). Hence, by adjusting $A$ to its appropriate value, the oscillation starting condition of the high-impedance resonator described in this article can be satisfied.

In the meantime, after introducing the feedback resistance, the gain of the circuit shown in Fig. 2 can be expressed as

$$
K_{V}=\frac{K_{0}}{1+K_{0} F}=\frac{K_{0}}{1+\left(-R_{3} /\left(R_{2}+R_{3}\right)\right) K_{0}},
$$

where $K_{0}$ is the circuit open-loop gain and $F$ is the feedback factor. When the feedback is so deep that $K_{0} F>>1$, then

$$
K_{V} \approx \frac{1}{F}=-\frac{\left(R_{2}+R_{3}\right)}{R_{3}}
$$

This indicates that when the condition of deep negative feedback is satisfied, the voltage amplification factor of the circuit only depends on the external resistor, and it is independent of the parameters of the transistor itself. Thus, the effects of the fluctuation of supply voltage and temperature changes on the resonance frequency and amplitude can be reduced, and the signal-to-noise ratio of the oscillation signal will be improved. ${ }^{(9-12)}$

\section{Simulation and Experiment Results}

In order to compare the performances of the improved circuit and traditional circuit, the gain and vibration wave of the two kinds of circuits are simulated using the PSPICE software. ${ }^{(13)}$ Figure 6 shows the gain curve of the improved circuit and the traditional circuit. Here, curve 1 represents the gain curve of the improved circuit, and curve 2 represents the gain curve of the traditional circuit. The gain simulation results show that the open-loop gain of the improved circuit achieves significant improvement, and the excitation performance of the high-impedance quartz vibrating-beam is greatly enhanced. Figure 7(a) shows the vibration wave of the traditional circuit, and Fig. 7(b) shows the vibration wave of the improved circuit. The vibrating simulation results show that the traditional circuit cannot excite the high impedance vibrating beam, whereas the highimpedance beam vibrates stably with the improved circuit.

The vibrating wave of the fabricated circuit is tested using the oscilloscope TDS3032B, and the test results are shown in Fig. 8. The test results are in accordance with the simulation results. The vibrating performance is evaluated using the Agilent frequency meter 53132A, and the test results are shown in Fig. 9. The test results show that the vibrating frequency bias stability of the system is less than $8 \mathrm{mHz}$ in about $40 \mathrm{~min}$. 


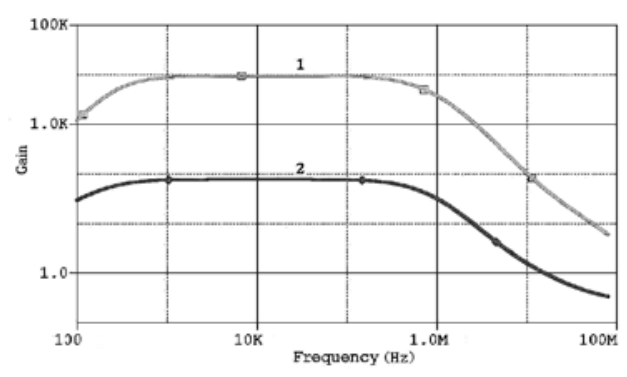

Fig. 6. Gains of the improved circuit and traditional circuit.

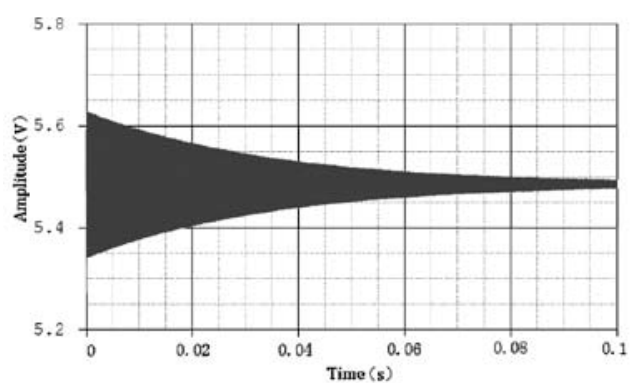

(a)

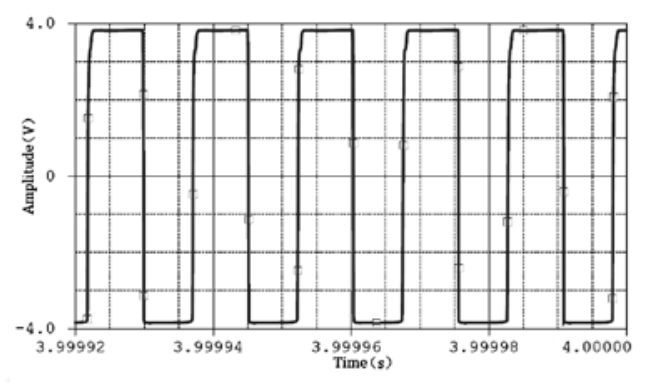

(b)

Fig. 7. Simulated vibrating wave. (a) Vibration of the traditional circuit. (b) Vibration of the improved circuit.

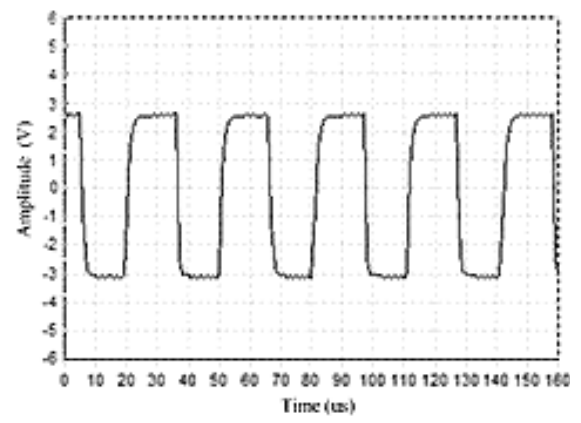

Fig. 8. Test vibrating wave of the resonant system with the fabricated circuit.

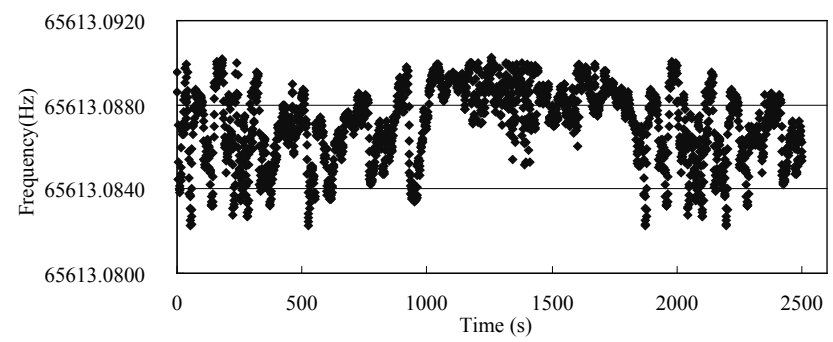

Fig. 9. Frequency test results of the resonant system with the fabricated circuit. 


\section{Conclusion and Summary}

On the basis of the analytical result of the traditional dual common-emitter series oscillator circuit, a high-performance oscillator circuit for a high-impedance quartz vibrating beam was designed and fabricated. The circuit was a series oscillator circuit with four common-emitter gain links and two negative feedbacks. The circuit had better excitation performance than the traditional dual common-emitter series oscillator circuit, and the high-impedance quartz vibrating beam vibrated stably with it. The evaluation results using the Agilent frequency meter 53132A showed that the vibrating frequency bias stability of the resonant system is less than $8 \mathrm{mHz}$. This work is significant for further development of the micromechanical quartz resonant accelerometer.

\section{Acknowledgements}

This work was supported by the National Natural Science Foundation of China (No. 61101021, No. 61101163), the Jiangsu Provincial Natural Science Foundation of China (No. BK2010401, No. BK2012739), the Specialized Research Fund for the Doctoral Program of Higher Education of China (No. 20100092110038), and the Guidance Foundation for Significant Research of Southeast University (No. 3222002105).

\section{References}

1 J. X. Liang, X. F. Li, H. S. Li, Y. F. Ni, K. Y. Li, L. B. Huang and T. Ueda: Jpn. J. Appl. Phys. 50 (2011) 06GM06-1.

2 F. Tan and X. H. Huang: Piezoelectrics Acoustooptics 28 (2006) 256 (in Chinese).

3 G. Rong, G. L. Liu, D. Li and Y. L. Wang: Sensor World 13 (2007) 15 (in Chinese).

4 W. Fu, F. Tan and X. H. Huang: Frequency Control Symposium (IEEE, Newport Beach, CA, 2010) pp. 443-445.

5 K. Fukuzawa, K. Hayakawa, N. Matsumura, S. Itoh and H. Zhang: Tribology Lett. 37 (2010) 497.

6 S. Kudo and S. Sugawara: Jpn. J. Appl. Phys. 41 (2002) 3439.

7 H. Toshiyoshi, H. Fujita and T. Ueda: J. Microelectromech. Syst. 4 (1995) 3.

8 J. K. A. Everard and K. Theodoropoulos: IEEE Frequency Control Symposium (IEEE, Miami, 2006) pp. 345-357.

9 F. Sthal, S. Galliou, N. Gufflet and M. Mourey: IEEE Trans. Ultrason. Ferroelectr. Freq. Control 52 (2005) 27.

10 F. Eichelbaum, R. Borngraber and J. Schroder: Rev. Sci. Instrum. 70 (1999) 2537.

11 A. Arnau: Sensors 8 (2008) 370.

12 B. Jakoby: IEEE Sens. J. 5 (2005) 1106.

13 A. A. Gubarev, A. V. Kosykh, S. A. Zavjavlov and A. N. Lepetaev: Proc. 2003 IEEE International Frequency Control Symposium and PDA Exhibition (IEEE, Tempa, Florida, 2003). 